\title{
DNA-image cytometry has promise for oral cancer detection
}

\section{Abstracted from}

\section{Ye X, Zhang J, Tan Y, Chen G, Zhou G.}

Meta-analysis of two computer-assisted screening methods for diagnosing oral precancer and cancer. Oral Oncol 2015; 51: 966-975.

Address for correspondence: Gang Zhou, Department of Oral Medicine, School and Hospital of Stomatology, Wuhan University, Wuhan, PR China. E-mail: gordonzhou@tom.com.

\section{Question: Which computer-assisted oral precancer and cancer diagnostic test has the highest accuracy?}

Data sources The Medline, Embase, PubMed, Elsevier and Web of Science databases and the reference lists of known primary and review papers were scanned for relevant citations.

Study selection Prospective and retrospective studies evaluating brush cytology were considered. Only computer-assisted methods that included histologically confirmed disease positive status were included. Data extraction and synthesis Data were extracted using a standardised form. Study quality was assessed by one reviewer using the quality assessment of diagnostic accuracy studies (QUADAS) checklist. Pooled sensitivity and specificity were calculated with $95 \% \mathrm{Cls}$ (confidence intervals) separately for each study. Likelihood and diagnostic odds ratios were also calculated along with a summary receiver-operating characteristic (SROC) curve analysis.

Results Thirteen studies (eight of OralCDx brush biopsy and five of DNA-image cytometry) reporting on 1981 oral mucosa lesions were included. OralCDx brush biopsy had a pooled sensitivity of $86 \%$ (95\% Cl; 81-90) and pooled specificity of $81 \%(95 \% \mathrm{Cl} ; 78-85)$. The pooled sensitivity and specificity of DNA-image cytometry were $89 \%$ (95\% Cl; 83-94) and 99\% (95\% Cl; 97-100). Diagnostic odds ratio estimates for OralCDx brush biopsy and DNA-image cytometry were 20.36 (95\% Cl; 2.72-152.67) and 446.08 (95\% Cl; $73.36-$ 2712.43), respectively. Study size was found to be closely related to heterogeneity among studies and analysis suggested publication bias in relation to OralCDx brush biopsy.

Conclusions The results of this meta-analysis suggest that DNA-image cytometry has a highly significant potential over OralCDx brush biopsy as an accurate and simple diagnostic tool for clinically suspected oral precancer and oral cancer.

\section{Commentary}

The subject of early detection of oral and oropharyngeal cancer has generated much discussion recently on several fronts. The mortality rates for oral cancer have not changed over the past several decades, and this is in stark contrast to cancers of the breast, colon and cervix. Additionally, there has been an increase in the incidence of HPV-related cancer of the oropharynx, and non-HPV related cancers of the tongue in young females with no known risk factors. ${ }^{1}$

This systematic review/meta-analysis examined differences between two diagnostic tests, namely the OralCDx and DNA-image cytometry. Systematic reviews of diagnostic tests present challenges apart from reviews dealing with therapeutic interventions. Some of these difficulties include identifying appropriate study designs, which is not the case for reviews of interventions which target randomised trials. Additionally, reviews of diagnostic tests include data that are in pairs, such as sensitivity/specificity or positive/ negative likelihood ratios which often vary in opposite directions. ${ }^{2}$ Reviews of trials more commonly deal with either single risk ratios or mean differences, summary estimates which are less complex to develop in meta-analyses.

This review started with a focused research question and a comprehensive search of several databases over a 34-year period. However, there was only a single author involved in abstract identification and data abstraction. Typically, dual, independent data abstraction is preferred to reduce bias. Neither non-English nor non-published studies were sought which can also bias results. While inclusion/exclusion criteria of studies were listed, there was no mention of the specific types of studies meeting the inclusion criteria, which should include a consecutive or random sample of patients. This is a concern, as the inclusion of case-control studies can exaggerate the accuracy of diagnostic tests. ${ }^{3}$ There was no mention of the study populations and settings which can also lead to spectrum bias due to differences in prevalence/severity of disease in targeted populations. However, risk of bias among included studies was thoroughly assessed with two tables examining many dimensions of quality in diagnostic test studies. In addition it appears that all patients in the included studies appeared to get both the index test and the reference standard, an important quality issue.

The authors did an excellent job in summarising the data with pooled sensitivity/specificity, DOR, AUC and Q-index. While sensitivity/specificity are useful indicators for how well a test works, they are limited when interpreting results for specific patients. This is where measures such as likelihood ratios (LR) and diagnostic odds ratios (DOR), along with area-under-the-curve (AUC) can be helpful. 
A useful rule of thumb is $+\mathrm{LR}>10$ and $-\mathrm{LR}<0.1$ indicate a strong ability to rule in and rule out disease, respectively. The + LR provided show that DNA-cytometry was effective at ruling in disease, whereas OralCDx was not. As the DOR represents the ratio of +LR /-LR, here we are looking for values $>100$ to demonstrate an effective test. With a pooled DOR of 446 for DNA-cytometry, the magnitude of the difference with a DOR of 20 for OralCDx cannot be ignored. This means that for DNA-cytometry, odds for positivity among patients with dysplasia/OSCC was 446 times higher than odds for positivity among patients without dysplasia/OSCC. ${ }^{4}$ This was also reflected in a higher value for AUC with DNA-cytometry.

The authors used the QUADAS tool for assessing quality of included studies and stated that the majority of included studies were of high quality. The quantity of evidence appeared adequate with 13 studies evaluating nearly 2000 lesions. There was marked inconsistency (statistical heterogeneity) among OralCDx studies and moderate inconsistency among DNA-cytometry papers. This is not surprising as diagnostic test studies often differ in many clinical components. Imprecision among effect estimates was also high as demonstrated by wide confidence intervals on DOR provided. This is most likely due to many of the individual studies having small sample sizes. Although assessment of publication bias was performed, results should be interpreted with caution due as typically 10 studies would be needed for each of the two types of tests being compared.

With the majority of oral cancers diagnosed at late stage disease, and the cost/invasiveness of biopsy, diagnostic tests with good overall utility have been lacking. Many of these tests have good sensitivity but poor specificity, resulting in many false positives leading to unnecessary biopsy, especially among low-risk populations. Despite some weaknesses with methods of this systematic review, DNAcytometry appears to offer promise in assisting practitioners with identifying potentially malignant lesions in early stage where they are more treatable and are associated with higher survival rates.

\section{Elliot Abt}

Department. of Dentistry, Illinois Masonic Medical Center, Chicago, Illinois, USA

1. Chi AC, Day TA, Neville BW. Oral cavity and oropharyngeal squamous cell carcinoma - an update. CA Cancer / Clin 2015; 65: 401-421.

2. Leeflang MM, Deeks J], Takwoingi Y, Macaskill P. Cochrane diagnostic test accuracy reviews. Syst Rev 2013; 2: 82.

3. QUADAS-2: a revised tool for the quality assessment of diagnostic accuracy studies. Ann Intern Med 2011; 155: 529-536.

4. Glas AS, Lijmer JG, Prins MH, Bonsel GJ, Bossuyt PM. The diagnostic odds ratio: a single indicator of test performance. J Clin Epidemiol 2003; 56: 1129-1135

Evidence-Based Dentistry (2015) 16, 106-107. doi:10.1038/sj.ebd.6401130 